\title{
Seeing the UN Sustainable Development Goals through Maps
}

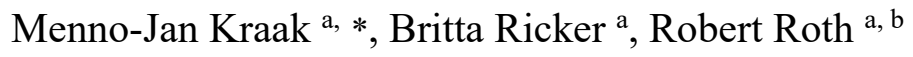 \\ a University of Twente/ITC,Department of Geo-Information Processing,m.j.kraak@utwente.nl,b.a.ricker@utwente.nl \\ ${ }^{b}$ University of Wisconsin-Madison, CartographyLab(USA), reroth@wisc.edu \\ * Corresponding author
}

Keywords: Sustainable Development Goals, cartographic principles, map design, social justice

Purpose: In this presentation, we announce a book project to map the United Nations (UN) Sustainable Development Goals (SDGs). The book project is a collaboration between the UN Cartographic Unit, Geospatial Information Section, and the International Cartographic Association (ICA), with layout and production completed by the University of Wisconsin Cartography Lab. The book provides a visual primer on basic and advanced cartographic design principles, demonstrating the power of mapping to engage with persistent social, economic, and environmental inequities while also illustrating the potential of maps to mislead and therefore reinforce these inequities. Target audiences include: policymakers and researchers collecting geospatial information on SDG indicators to improve data policy and products; analysts and designers utilizing geospatial information to improve maps and graphics of the SDGs; and the general public to promote awareness and dialogue about the most pressing challenges facing our planet. In the following, we describe background on the SDGs, book organization, and development process.

Background: The UN identifies 17 SDGs in an effort to collectively address global disparities [1], with goals including dimensions of social inclusion, economic growth, and environmental sustainability (Table 1). While ambitious and imperfect, the SDGs provide a shared vision for eliminating global inequities and affecting local change through collective accountability and governance [2-4]. Each of the seventeen goals has a set of targets and associated indicators to assess global disparities and monitor progress towards the SDGs, providing a framework for shared action [5]. Of the 232 total indicators identified by the UN, 93 currently are "Tier 1" with data produced regularly by at least $50 \%$ of the world's nations. Thus, only $\sim 40 \%$ of the SDG indicators are mappable on a global scale, and many of the currently compiled datasets are wrought with redundancies and uncertainties.

Cartography has an important role to play in communicating SDG indicator data, and thus in our understanding of the SDGs and their impacts. For instance, the array of SDG indicators are prescribed at different levels of

\begin{tabular}{|c|l|}
\hline \multicolumn{2}{|c|}{ Table 1: UN Sustainable Development Goals } \\
\hline 1 & No Poverty \\
\hline 2 & Zero Hunger \\
\hline 3 & Good Health \& Well-being \\
\hline 4 & Quality Education \\
\hline 5 & Gender Equity \\
\hline 6 & Clean Water \& Sanitation \\
\hline 7 & Affordable \& Clean Energy \\
\hline 8 & Decent Work \& Economic Growth \\
\hline 9 & Industry, Innovation, \& Infrastructure \\
\hline 10 & Reduced Inequalities \\
\hline 11 & Sustainable Cities \& Communities \\
\hline 12 & Responsible Production \& Consumption \\
\hline 13 & Climate Action \\
\hline 14 & Life Below Water \\
\hline 15 & Life on Land \\
\hline 16 & Peace, Justice, \& Strong Institutions \\
\hline 17 & Partnerships for These Goals \\
\hline
\end{tabular}
measurement (e.g., nominal, ordinal, ratio) and use different strategies for normalization (e.g., none, proportions, rates, indices) [6]. Cartography offers general guidelines for transforming and mapping existing indicators and new ones as they become available, while remaining adaptable to specific contexts and needs. Embedding cartographic knowledge into the SDGs also overcomes perceived shortfalls of open data and neogeographic interfaces that enables the public to create powerful, yet potentially misleading maps. The book project reported here translates the SDG indicators into the language of cartography, identifying opportunities and pitfalls for mapping the SDGs, and ultimately inspiring better cartography to assess and monitor the SDGs.

Organization: The book contains $\sim 75$ unique topics organized into four broad sections based on priorities identified by the collaborative core team:

(1) SDGs and Geospatial Temporal Data: Topics include overviews of the SDGs, targets, indicators, and data sources; introduction to locational, attribute, and temporal dimensions of data; fundamentals of geographic linework such as dimensionality; and statistical considerations such as enumeration, levels of measurement, data type conversations and data classification.

(2) Cartographic Design Principles: Topics include map design planning and workflows; scale and generalization; map projections; symbolization and color; typography and toponymy; and visual hierarchy and layout.

(3) Map and Diagram Techniques: Topics include thematic map types such as choropleth, dot density, isoline, and proportional symbols; diagrams accompanying maps such as bar graphs, line graphs, and pie charts; and advanced representation techniques such as cartograms, bivariate maps, and animated maps.

(4) Map Use Environments: Topics include considerations for audience and user needs; interactivity and new media; designing for the web and mobile platforms; storytelling and presentation; and map evaluation.

Each topic is composed as a bi-fold tabloid spread (resulting in a $\sim 150$ page booklet) and includes 250 words of explanatory text on the treated cartographic principle and one or several map examples demonstrating sound versus 
problematic cartographic design. Visual examples make use of SDG indicator datasets and include descriptive captions, with each two-page panel both introducing a cartographic design concept and providing a visual interpretation of the geography of the associated SDG, and the inequities and alternatives therein (see Figure 1 for a working design template).

Process: The core team of contributors have completed the foundational set of "Basics" entries that provide the backbone for the book. We then will solicit ICA Commissions to contribute one of three kinds of entries for deeper discussion, based on the SDG posters created in 2017-2018: (1) "Best Practice" entries explaining well-established cartographic design principles and associated common missteps (e.g., use of Mercator for choropleth maps; use of stoplight metaphors inaccessible to color vision deficiency); (2) "How to" entries providing conceptual or technical design guidance on new and emerging design opportunities (Figure 1); and (3) "Role of" entries explicitly relating cartography to the SDGs and the broader mission of the UN. The core team will seek a final solicitation from ICA Commissions after this presentation at the ICC 2019 in Tokyo, infilling any remaining gaps to ensure a publication schedule by Q4 of 2019. The book will be available as a printed softcover booklet and a download pdf that can be repackaged as a slide deck.

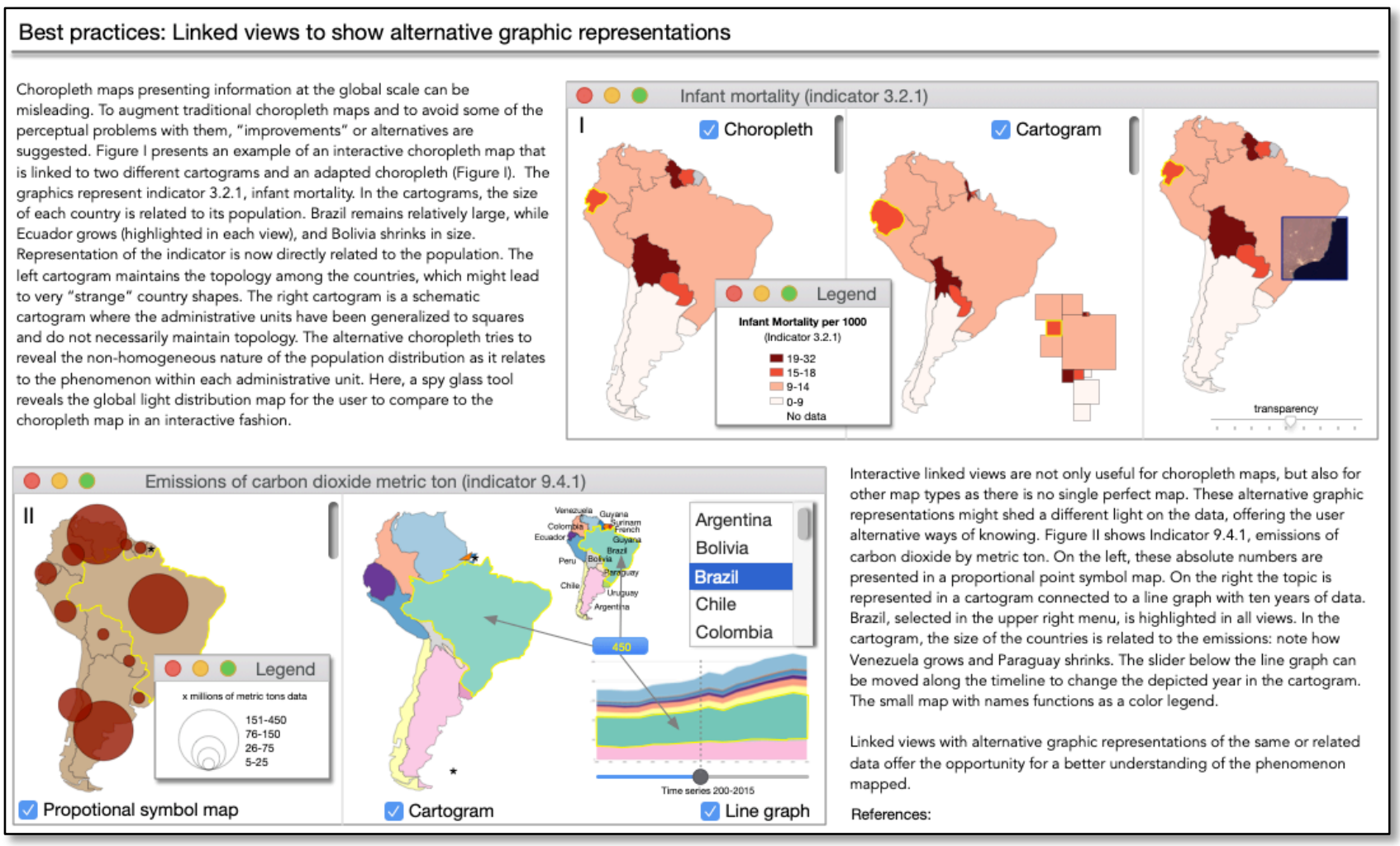

Figure 1. Example design template for "Linked views to show alternative graphic representations".

\section{References:}

1. United Nations. Sustainable Development Goal Indicators. 2018 [accessed 30 December 2018]; Available from: https://unstats.un.org/sdgs/.

2. Biermann, F., N. Kanie, and R.E. Kim, Global governance by goal-setting: The novel approach of the UN Sustainable Development Goals. Curr. Opin. Environ. Sustain., 2017. 26-27: p. 26-31.

3. Griggs, D., et al., Policy: Sustainable development goals for people and planet. Nature, 2013. 495(305-307).

4. Fukuda-Parr, S., From the Millennium Development Goals to the Sustainable Development Goals: Shifts in purpose, concept and politics of global goal setting for development. Gend. Dev., 2016. 24: p. 43-52.

5. United Nations. SDG Global Indicators Global Database. 2018 [accessed 30 December 2018]; Available from: https://unstats.un.org/sdgs/indicators/database/.

6. Kraak, M.-J., B. Ricker, and Y. Engelhardt, Challenges of mapping Sustainable Development Goals indicators data. International Journal of Geo-Information, 2018. 7: p. 482. 